Tumor ablation and volumetric optoacoustic monitoring with a short-pulsed laser source

\author{
Periyasamy, Vijitha ; Özsoy, Çağla ; Reiss, Michael ; Deán-Ben, Xosé Luís ; Razansky, Daniel
}

DOI: https://doi.org/10.1117/12.2531536

Posted at the Zurich Open Repository and Archive, University of Zurich ZORA URL: https://doi.org/10.5167/uzh-200698

Conference or Workshop Item

Published Version

Originally published at:

Periyasamy, Vijitha; Özsoy, Çağla; Reiss, Michael; Deán-Ben, Xosé Luís; Razansky, Daniel (2019). Tumor ablation and volumetric optoacoustic monitoring with a short-pulsed laser source. In: Opto-Acoustic Methods and Applications in Biophotonics, Munich, 23 June 2019 - 27 June 2019, Spie.

DOI: https://doi.org/10.1117/12.2531536 


\section{Tumor ablation and volumetric optoacoustic monitoring with a short- pulsed laser source}

Periyasamy, Vijitha, Özsoy, Çağla, Reiss, Michael, DeánBen, Xosé Luís, Razansky, Daniel

Vijitha Periyasamy, Çağla Özsoy, Michael Reiss, Xosé Luís Deán-Ben, Daniel Razansky, "Tumor ablation and volumetric optoacoustic monitoring with a short-pulsed laser source," Proc. SPIE 11077, Opto-Acoustic Methods and Applications in Biophotonics IV, 1107708 (19 July 2019); doi: 10.1117/12.2531536 


\title{
Tumor ablation and volumetric optoacoustic monitoring with a short-pulsed laser source
}

\author{
Vijitha Periyasamy ${ }^{l}$, Çăgla Özsoy ${ }^{2,3}$, Michael Reiss ${ }^{2,3}$, Xosé Luís Deán-Ben ${ }^{2,3}$, and Daniel Razansky ${ }^{1,2,3, *}$ \\ ${ }^{1}$ Institute for Biological and Medical Imaging (IBMI), Helmholtz Center Munich and Technical University of Munich, Neuherberg, Germany. \\ ${ }^{2}$ Faculty of Medicine and Institute of Pharmacology and Toxicology, University of Zurich, Switzerland. \\ ${ }^{3}$ Institute for Biomedical Engineering and Department of Information Technology and Electrical Engineering, ETH Zurich, Switzerland. \\ * Corresponding author: daniel.razansky@uzh.ch
}

\begin{abstract}
Laser ablation (LA) represents a minimally invasive intervention that is gaining acceptance for the treatment of different types of cancer, leading to important advantages such as less pain and shorter recovery time. Accurate monitoring of ablation progression is crucial to prevent damage of non-cancerous tissues and optimize the outcome of the intervention. To this end, imaging techniques such as ultrasound, computed tomography or magnetic resonance imaging have been used for monitoring LA. However, these techniques feature important drawbacks such as the need of contrast agents, poor spatio-temporal resolution or high cost. Optoacoustics (OA, photoacoustic) has recently been shown to provide unique properties to monitor thermal treatments. Herein, we demonstrate the feasibility of optoacoustic laser-ablation (OLA) monitoring in a murine breast tumor model using a single short-pulsed $1064 \mathrm{~nm}$ laser source. The effect of irradiation was volumetrically tracked with the OA images acquired with a 256-element spherical array. Structural damage of the tissue was clearly seen during the LA procedure.
\end{abstract}

\section{Introduction}

Cancer treatments by laser ablation (LA) are gaining popularity as a viable alternative to tumor resection ${ }^{1-3}$. LA is considered a minimally invasive intervention causing less pain and involving shorter recovery time than standard surgery ${ }^{2,4,5}$. Monitoring of the LA procedure is essential to determine the end point of irradiation and optimize the treatment outcome. Nd:YAG laser sources operated at $1064 \mathrm{~nm}$ are commonly used to provide irradiation energy, which can penetrate a few millimeters to centimeters into biological tissues. Some of the effects induced by laser exposure are tissue heating, tissue removal, thermal injury, tissue vaporization, melting, ejection and pyrolysis ${ }^{3}$. These and other effects can be visualized using imaging modalities such as computed tomography, ultrasound and magnetic resonance imaging. However, these techniques have drawbacks such as the need for contrast agents, poor resolution or high costs and logistical complexity.

Optoacoustic (OA, photoacoustic) imaging has been widely employed for small animal imaging ${ }^{6-16}$ and has additionally been demonstrated to provide unique advantages for monitoring different types of thermal treatments ${ }^{17-}$ ${ }^{25}$. As examples, real-time OA imaging during radiofrequency (RF) ablation of ex vivo tissues has being shown ${ }^{26}$. Validation of the OA images of RF ablated tissues was further showcased ${ }^{27}$. Additionally, OA has been used to monitor ultrasound heating ${ }^{28}$, laser thermal treatments ${ }^{21,29}$ or cryoablation ${ }^{30}$. In this work, we demonstrate the feasibility of optoacoustic laser-ablation (OLA), which is simultaneous OA imaging and LA of a murine breast tumor model in-vivo using the same $1064 \mathrm{~nm}$ short-pulsed laser source.

Opto-Acoustic Methods and Applications in Biophotonics IV, edited by Vasilis Ntziachristos,

Roger Zemp, Proc. of SPIE-OSA Vol. 11077, 1107708 • (c) 2019 SPIE-OSA

CCC code: $1605-7422 / 19 / \$ 21 \cdot$ doi: $10.1117 / 12.2531536$ 


\section{Materials and Methods}

\subsection{Experimental set-up.}

The experimental system used for laser ablation of tumors is depicted in Fig. 1. The $1064 \mathrm{~nm}$ output beam from Nd:YAG laser (Innolas, GmbH, Krailling, Germany) was delivered into a subcutaneous mouse tumor. A mirror (PF1003-P01P, Thorlabs, Newton, NJ, USA) and a diverging lens (LC1715-C, Thorlabs, Newton, NJ, USA) were used to guide and diverge the laser beam to illuminate the sample with a beam diameter of $\sim 1 \mathrm{~cm}$, thus covering the entire tumor. The light beam provided $10 \mathrm{~W}$ of energy deposited on the sample at a pulse repetition of $100 \mathrm{~Hz}$. The OA pressure waves generated during the ablation procedure were acquired using a 256 element spherical array transducer with a center frequency of $4 \mathrm{MHz}$ and 100\% detection bandwidth (Imasonic SaS Voray, France) ${ }^{31}$. A data acquisition unit (Falkenstein Mikrosysteme GmbH, Taufkirchen, Germany) with a sampling frequency of 40 megasamples/s was used to amplify and digitize the signals. Volumetric images of a region with size $20 \times 20 \times 20 \mathrm{~mm}^{3}$ (200x200x200 voxels) were reconstructed with a standard back-projection algorithm considering an average speed of sound of $1500 \mathrm{~m} / \mathrm{s}$. Image reconstruction and post-processing was done with a self-developed MATLAB software based code, executed on a personal computer (Intel xeon E5-1630 3.7 GHz processor, 16 GB RAM, 64 bit windows 10 operating system).

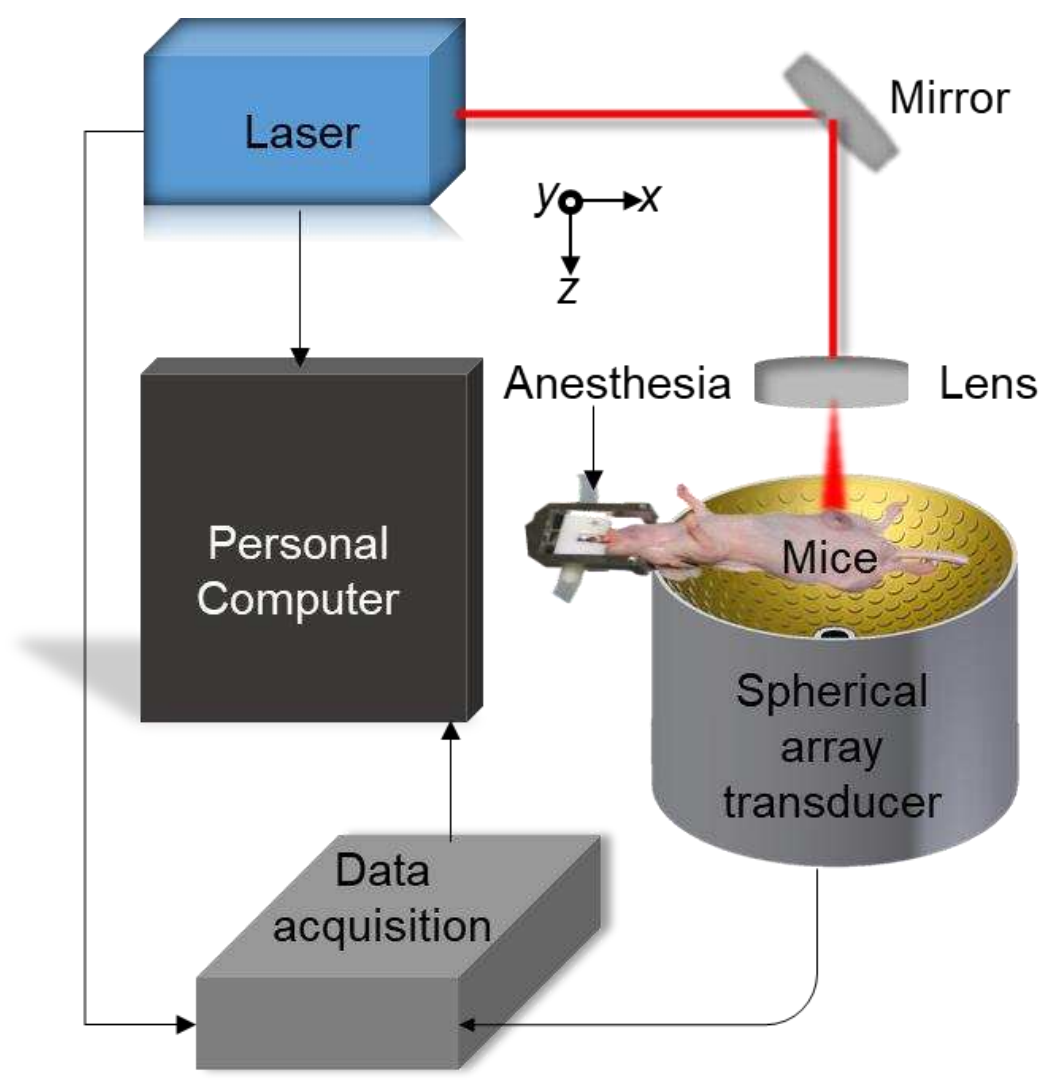

Fig 1: Experimental set-up for laser ablation of a murine tumor model. 


\subsection{Ablation experiment.}

A Athymic Nude Foxn1 mouse (Envigo, Huntingdon, UK) mouse was injected with 4T1 tumor cells in the mammary fat pad. The induced tumor was ablated 10 days after cell inoculation. For this, the mouse was placed in supine position on top of an agar block used to fill the volume enclosed by the spherical array to guarantee acoustic coupling. Thermocouples were inserted into the core of the tumor and periphery of the tumor to monitor the temperature rise during the OLA. 30 minutes before the ablation the mouse received $0.1 \mathrm{mg} / \mathrm{kg}$ Buprenorphin s.c. The mouse was kept under isoflurane anesthesia during the LA procedure and euthanized immediately after. The experiment was performed in accordance with institutional guidelines of Helmholtz Zentrum München and the government of Upper Bavaria.

\section{Results}

The disruption of the tissue structure during ablation can be recognized by inspecting the maximum intensity projections (MIPs) of the reconstructed OA images shown in Fig. 2(a). Five representative time points during the $130 \mathrm{~s}$ ablation procedure are shown. The MIPs along the lateral ( $x z$ plane) and axial ( $x y$ plane) directions are displayed in the top and bottom panels, respectively. The projections are normalized by the maximum intensity of the volume in the entire image sequence. The intensity of OA signal increases during initial heating and then reduces over further irradiation. Initial rise in temperature could be attributed to the absorbance by the blood in the blood vessels which are destroyed on heating beyond $30 \mathrm{~s}$ exposure. The trend of initial rise and drop of OA signal shown here is similar to the trend shown by Felm et al. for endovenous laser therapy ${ }^{32}$. Figs. 2(b) and 2(c) are photographs of the tumor taken before and after ablation, respectively. The reduced redness and increased volume of the post-ablated tumor validates the induced tissue damage. The clearly observed changes in the OA images during laser ablation may serve to identify the end points of the ideal ablation procedure.
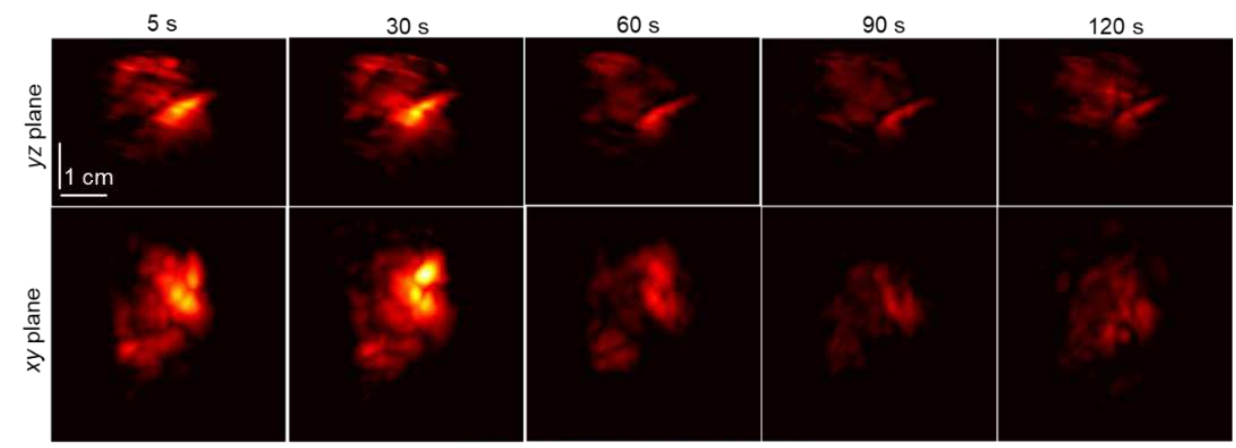

(a)

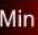
Max
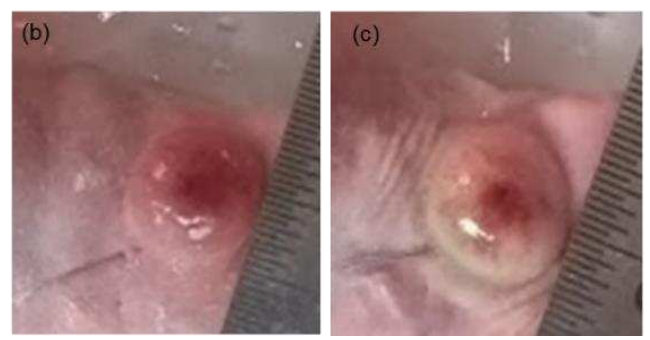

Fig 2: (a) Maximum intensity projections (MIPs) of the reconstructed optoacoustic images along the $x$ ( $y z$ plane) and $z$ ( $x y$ plane) directions at 5, 30, 60, 90 and $120 \mathrm{~s}$. (b) Photograph of the tumor before ablation. (c) Photograph of the tumor after ablation. 


\section{Discussion and Conclusions.}

One of the most important challenges of LA is the determination of the end point of energy deposition. Insufficient heating leads to recurrence of the tumor while overdose of irradiation causes damage of the surrounding healthy tissue. Hence, monitoring of thermal doses in real-time is a primary need during LA. Herein, the feasibility of OLA, OA monitoring of LA using the same short-pulsed laser source was demonstrated. The effect of irradiation was volumetrically tracked and the structural damage induced in the tissue was clearly seen during the LA procedure. OA may additionally serve to determine that (1) the ablation zone covers the entire area of the tumor and (2) sufficient exposure at the desired temperature is achieved, which however was not demonstrated in this study. The presented results anticipate the applicability of OA as a LA monitoring tool in the clinical setting. As future steps, we aim to automatically shape the beam according to the size of the tumor such that the temperature is maintained below vaporization at all points and improve the image reconstruction procedures to better visualize the internal structures. Also, accurate extraction of the temperature distribution in the tumor volume remains a major challenge for OLA that we also aim to address.

\section{Acknowledgement}

This project received funding from the German Research Foundation (DFG) under Research Grant RA1848/5-1.

\section{References}

[1] Schwartzberg, B., Lewin, J., Abdelatif, O., Bernard, J., Bu-Ali, H., Cawthorn, S., Chen-Seetoo, M., Feldman, S., Govindarajulu, S., Jones, L., Juette, A., Kavia, S., Maganini, R., Pain, S., Shere, M., Shriver, C., Smith, S., Valencia, A., Whitacre, E., et al., "Phase 2 Open-Label Trial Investigating Percutaneous Laser Ablation for Treatment of Early-Stage Breast Cancer: MRI, Pathology, and Outcome Correlations,” Ann. Surg. Oncol. 25(10), 2958-2964 (2018).

[2] Schena, E., Saccomandi, P. and Fong, Y., "Laser Ablation for Cancer: Past, Present and Future,” J. Funct. Biomater. 8(2), 19 (2017).

[3] Vogel, A. and Venugopalan, V., "Mechanisms of Pulsed Laser Ablation of Biological Tissues," Chem. Rev. 103(2), 577644 (2003).

[4] Vogel, A. and Venugopalan, V., "Pulsed laser ablation of soft biological tissues," [Optical-Thermal Response of LaserIrradiated Tissue], Springer, 551-615 (2010).

[5] Chu, K. F. and Dupuy, D. E., "Thermal ablation of tumours: biological mechanisms and advances in therapy,” Nat. Rev. Cancer 14(3), 199 (2014).

[6] Hu, S., “Listening to the Brain With Photoacoustics,” IEEE J. Sel. Top. Quantum Electron. 22(3), 6800610 (2016).

[7] Sangha, G. S., Phillips, E. H. and Goergen, C. J., "In vivo photoacoustic lipid imaging in mice using the second nearinfrared window," Biomed Opt Express 8(2), 1 Feb 2017, 736-742 (2017).

[8] Merčep, E., Herraiz, J. L., Deán-Ben, X. L. and Razansky, D., "Transmission-reflection optoacoustic ultrasound (TROPUS) computed tomography of small animals,” Light Sci. Appl. (2019).

[9] Deán-Ben, X. L., López-Schier, H. and Razansky, D., “Optoacoustic micro-tomography at 100 volumes per second,” Sci. Rep. 7(1), 6850 (2017).

[10] Deán-Ben, X. L., Merčep, E. and Razansky, D., "Hybrid-array-based optoacoustic and ultrasound (OPUS) imaging of biological tissues,” Appl. Phys. Lett. 110(20), 203703 (2017).

[11] Ron, A., Deán-Ben, X. L., Reber, J., Ntziachristos, V. and Razansky, D., "Characterization of Brown Adipose Tissue in a Diabetic Mouse Model with Spiral Volumetric Optoacoustic Tomography,” Mol. Imaging Biol., 1-6 (2018).

[12] Wang, L. V. and Yao, J., “A practical guide to photoacoustic tomography in the life sciences,” Nat. Methods 13(8), 627638 (2016). 
[13] Meng, L., Deschaume, O., Larbanoix, L., Fron, E., Bartic, C., Laurent, S., der Auweraer, M. and Glorieux, C., "Photoacoustic temperature imaging based on multi-wavelength excitation," Photoacoustics 13, 33-45 (2018).

[14] Joseph, F. K., Chinni, B., Channappayya, S. S., Pachamuthu, R., Dogra, V. S. and Rao, N., "Two sided residual refocusing for acoustic lens based photoacoustic imaging system," Phys. Med. Biol. 63(13), 13NT03 (2018).

[15] Schellenberg, M. W. and Hunt, H. K., "Hand-held optoacoustic imaging: A review," Photoacoustics 11, 14-27 (2018).

[16] Sivasubramanian, K., Periyasamy, V., Wen, K. K. and Pramanik, M., "Optimizing light delivery through fiber bundle in photoacoustic imaging with clinical ultrasound system: Monte Carlo simulation and experimental validation,” J. Biomed. Opt. 22(4), 41008 (2017).

[17] Kim, H., Jo, G. and Chang, J. H., "Ultrasound-assisted photothermal therapy and real-time treatment monitoring,” Biomed. Opt. Express 9(9), 4472 (2018).

[18] Nguyen, V. P., Oh, J., Park, S. and Wook Kang, H., "Feasibility of photoacoustic evaluations on dual-thermal treatment of ex vivo bladder tumors," J. Biophotonics 10(4), 577-588 (2017).

[19] Rebling, J., Landa, F. J. O., Deán-Ben, X. L., Douplik, A. and Razansky, D., "Integrated catheter for simultaneous radio frequency ablation and optoacoustic monitoring of lesion progression," Opt. Lett. 43(8), 1886-1889 (2018).

[20] Pang, G. A., Bay, E., DEÁN-BEN, X. L. and Razansky, D., “Three-dimensional optoacoustic monitoring of lesion formation in real time during radiofrequency catheter ablation,” J. Cardiovasc. Electrophysiol. 26(3), 339-345 (2015).

[21] Bay, E., Douplik, A. and Razansky, D., "Optoacoustic monitoring of cutting efficiency and thermal damage during laser ablation,” Lasers Med. Sci. 29(3), 1029-1035 (2014).

[22] Petrova, E., Ermilov, S., Su, R., Nadvoretskiy, V., Conjusteau, A. and Oraevsky, A., "Using optoacoustic imaging for measuring the temperature dependence of Grüneisen parameter in optically absorbing solutions," Opt. Express 21(21), 25077-25090 (2013).

[23] Chitnis, P. V, Brecht, H.-P. F., Su, R. and Oraevsky, A. A., "Feasibility of optoacoustic visualization of high-intensity focused ultrasound-induced thermal lesions in live tissue," J. Biomed. Opt. 15(2), 21313 (2010).

[24] Kim, J., Choi, W., Park, E.-Y., Kang, Y., Lee, K. J., Kim, H. H., Kim, W. J. and Kim, C., "Real-time Photoacoustic Thermometry Combined with Clinical Ultrasound Imaging and High Intensity Focused Ultrasound," IEEE Trans. Biomed. Eng. (2019).

[25] Larin, K. V, Larina, I. V and Esenaliev, R. O., "Monitoring of tissue coagulation during thermotherapy using optoacoustic technique," J. Phys. D. Appl. Phys. 38(15), 2645 (2005).

[26] Pang, G. A., Bay, E., Deán-Ben, X. L. and Razansky, D., “Optoacoustic monitoring of real-time lesion formation during radiofrequency catheter ablation," Photons Plus Ultrasound Imaging Sens. 2015 9323, 932308, International Society for Optics and Photonics (2015).

[27] Bouchard, R., Dana, N., Di Biase, L., Natale, A. and Emelianov, S., "Photoacoustic characterization of radiofrequency ablation lesions," Photons Plus Ultrasound Imaging Sens. 2012 8223(February 2012), 82233K, International Society for Optics and Photonics (2012).

[28] Landa, F. J. O., Penacoba, S. R., de Espinosa, F. M., Razansky, D. and Deán-Ben, X. L., “Four-dimensional optoacoustic monitoring of tissue heating with medium intensity focused ultrasound," Ultrasonics (2018).

[29] Bay, E., Deán-Ben, X. L., Pang, G. A., Douplik, A. and Razansky, D., "Real-time monitoring of incision profile during laser surgery using shock wave detection," J. Biophotonics 8(1-2), 102-111 (2015).

[30] Petrova, E., Liopo, A., Nadvoretskiy, V. and Ermilov, S., "Imaging technique for real-time temperature monitoring during cryotherapy of lesions,” J. Biomed. Opt. 21(11), 116007 (2016).

[31] Dean-Ben, X. L., Razansky, D., Deán-Ben, X. L., Razansky, D., Dean-Ben, X. L. and Razansky, D., "Portable spherical array probe for volumetric real-time optoacoustic imaging at centimeter-scale depths," Opt. Express 21(23), 28062-28071 (2013).

[32] Fehm, T. F., Deán-Ben, X. L., Schaur, P., Sroka, R. and Razansky, D., "Volumetric optoacoustic imaging feedback during endovenous laser therapy-an ex vivo investigation," J. Biophotonics 9(9), 934-941 (2016). 